\title{
SOME REMARKS ON DUALITY IN $S^{3}$
}

\author{
IAN R. PORTEOUS \\ Department of Mathematical Sciences \\ University of Liverpool \\ Liverpool, L69 3BX, UK \\ e-mail: porteous@liv.ac.uk
}

\begin{abstract}
In this paper we review some of the concepts and results of V. I. Arnol'd [1] for curves in $S^{2}$ and extend them to curves and surfaces in $S^{3}$.

1. Introduction. In [1] Arnol'd introduces the concepts of the dual curve and the derivative curve of a smooth $\left(=C^{\infty}\right)$ embedded curve in $S^{2}$. In particular he shows that the evolute or caustic of such a curve is the dual of the derivative. The dual curve is just the unit normal curve, while the derivative is the unit tangent curve. The definitions extend in an obvious way to curves on $S^{2}$ with ordinary cusps. Then one proves easily that where a curve has an ordinary geodesic inflection the dual has an ordinary cusp and vice versa. Since the derivative of a curve without cusps clearly has no cusps it follows that the caustic of such a curve has no geodesic inflections. This prompts investigating what kind of singularity a curve must have for its caustic to have a geodesic inflection, by analogy with the classical construction of de l'Hôpital, see [2], pages 24-26.

In the second and third parts of the paper the definitions of Arnol'd are extended to surfaces in $S^{3}$ and to curves in $S^{3}$.

The notations are those of Porteous [2], differentiation being indicated by subscripts.
\end{abstract}

2. Curves in $S^{2}$. Let $\mathbf{r}$ be a parametrised smooth curve on $S^{2}$. Then

$$
\begin{gathered}
\mathbf{r} \cdot \mathbf{r}=1, \quad \mathbf{r} \cdot \mathbf{r}_{1}=0 \\
\mathbf{r} \cdot \mathbf{r}_{2}+\mathbf{r}_{1} \cdot \mathbf{r}_{1}=0, \quad \mathbf{r} \cdot \mathbf{r}_{3}+3 \mathbf{r}_{1} \cdot \mathbf{r}_{2}=0 \\
\mathbf{r} \cdot \mathbf{r}_{4}+4 \mathbf{r}_{1} \cdot \mathbf{r}_{3}+3 \mathbf{r}_{2} \cdot \mathbf{r}_{2}=0 \\
\text { and } \quad \mathbf{r} \cdot \mathbf{r}_{5}+5 \mathbf{r}_{1} \cdot \mathbf{r}_{4}+10 \mathbf{r}_{2} \cdot \mathbf{r}_{3}=0
\end{gathered}
$$

1991 Mathematics Subject Classification: Primary 53A04.

The paper is in final form; although it would not be submitted for publication in any other journal, much of it is the basis of a fresh chapter in the author's current revision of Geometric Differentiation. 
Then either $\mathbf{r}$ is regular at a point, that is $\mathbf{r}_{1} \neq 0$, in which case $\mathbf{r} \cdot \mathbf{r}_{2} \neq 0$, implying that $\mathbf{r}_{2} \neq 0$, or $\mathbf{r}_{1}=0$. In the latter case

$$
\begin{gathered}
\mathbf{r} \cdot \mathbf{r}_{2}=0, \quad \mathbf{r} \cdot \mathbf{r}_{3}=0 \\
\mathbf{r} \cdot \mathbf{r}_{4}+3 \mathbf{r}_{2} \cdot \mathbf{r}_{2}=0 \text { and } \mathbf{r} \cdot \mathbf{r}_{5}+10 \mathbf{r}_{2} \cdot \mathbf{r}_{3}=0 .
\end{gathered}
$$

Suppose that in this case also $\mathbf{r}_{2} \neq 0$. There are then two cases. Either $\mathbf{r}_{3}$ is linearly independent of $\mathbf{r}_{2}$, and the curve has an ordinary cusp, or $\mathbf{r}_{3}$ is a multiple of $\mathbf{r}_{2}$, say $\mathbf{r}_{3}=3 \lambda \mathbf{r}_{2}$, with $\mathbf{r}_{4}$ not a multiple of $\mathbf{r}_{2}$, since $\mathbf{r} \cdot \mathbf{r}_{4}=-3 \mathbf{r}_{2} \cdot \mathbf{r}_{2} \neq 0$. What about $\mathbf{r}_{5}$ ? Suppose that $\mathbf{r}_{5}=\beta \mathbf{r}_{2}+\gamma \mathbf{r}_{4}$. Then

$$
-30 \lambda \mathbf{r}_{2} \cdot \mathbf{r}_{2}=-3 \gamma \mathbf{r}_{2} \cdot \mathbf{r}_{2}
$$

implying that $3(\gamma-10 \lambda) \mathbf{r}_{2} \cdot \mathbf{r}_{2}, \neq 0$ unless $\gamma=10 \lambda$.

So either $\mathbf{r}_{5}=\beta \mathbf{r}_{2}+10 \lambda \mathbf{r}_{4}$, for some $\beta \in \mathbf{R}$, or $\mathbf{r}_{5}$ is linearly independent of $\mathbf{r}_{2}$ and $\mathbf{r}_{4}$. In the latter case we say that $\mathbf{r}$ has an ordinary rhamphoid cusp, while in the former case we say that it has a special rhamphoid cusp.

Now let the curve $\mathbf{r}$ be oriented and co-oriented. The orientation determines the unique unit tangent curve or derivative curve $\mathbf{t}$ while the co-orientation determines the unique unit normal curve or dual curve $\mathbf{n}$, with $\mathbf{n} \cdot \mathbf{r}=0$ and $\mathbf{n} \cdot \mathbf{r}_{1}=0$. The dual of the dual is the curve $-\mathbf{r}$ antipodal to $\mathbf{r}$ on the sphere. For, since $\mathbf{n} \cdot \mathbf{r}=0$ it follows at once that $\mathbf{n} \cdot \mathbf{r}_{1}=0$ if and only if $\mathbf{r} \cdot \mathbf{n}_{1}=0$.

Geodesics on $S^{2}$ are great circles, and a regular curve $\mathbf{r}$ on $S^{3}$ is said to have an ordinary geodesic inflection at a point if at that point the vectors $\mathbf{r}, \mathbf{r}_{1}$ and $\mathbf{r}_{2}$ are coplanar, but $\mathbf{r}_{3}$ does not lie in that plane.

Proposition 1. Where a curve $\mathbf{r}$ on $S^{2}$ has an ordinary geodesic inflection the dual curve $\mathbf{n}$ has an ordinary cusp, and vice versa.

Pro of. Since $\mathbf{n} \cdot \mathbf{r}=0$ and $\mathbf{n} \cdot \mathbf{r}_{1}=0$ we have $\mathbf{n}_{1} \cdot \mathbf{r}=0$,

$$
\begin{array}{rll}
\mathbf{n} \cdot \mathbf{r}_{2}+\mathbf{n}_{1} \cdot \mathbf{r}_{1}=0 & \text { and } & \mathbf{n}_{1} \cdot \mathbf{r}_{1}+\mathbf{n}_{2} \cdot \mathbf{r}=0, \\
\mathbf{n} \cdot \mathbf{r}_{3}+2 \mathbf{n}_{1} \cdot \mathbf{r}_{2}+\mathbf{n}_{2} \cdot \mathbf{r}_{1}=0 & \text { and } & \mathbf{n}_{1} \cdot \mathbf{r}_{2}+2 \mathbf{n}_{2} \cdot \mathbf{r}_{1}+\mathbf{n}_{3} \cdot \mathbf{r}=0 .
\end{array}
$$

Moreover at a point of regularity of the curve $\mathbf{n}, \mathbf{r}$ and $\mathbf{r}_{1}$ form a basis for $\mathbf{R}^{3}$. Where $\mathbf{r}$ has an ordinary geodesic inflection then also $\mathbf{n} \cdot \mathbf{r}_{2}=0$. Then not only $\mathbf{n}_{1} \cdot \mathbf{n}=0$ and $\mathbf{n}_{1} \cdot \mathbf{r}=0$ but also $\mathbf{n}_{1} \cdot \mathbf{r}_{1}=0$, implying that $\mathbf{n}_{1}=0$. However $\mathbf{n} \cdot \mathbf{r}_{3} \neq 0$, implying that $\mathbf{n}_{2} \cdot \mathbf{r}_{1} \neq 0$ and $\mathbf{n}_{3} \cdot \mathbf{r} \neq 0$, and therefore that $\mathbf{n}_{2} \neq 0$ and $\mathbf{n}_{3} \neq 0$, while, since $\mathbf{n}_{2} \cdot \mathbf{r}=0$ but $\mathbf{n}_{3} \cdot \mathbf{r} \neq 0, \mathbf{n}_{3}$ is not a multiple of $\mathbf{n}_{2}$. That is the dual curve $\mathbf{n}$ has an ordinary cusp.

The reverse implication is proved in similar fashion.

It may happen that at some point of a curve $\mathbf{r}$ not only $\mathbf{r}_{1}=0$, with $\mathbf{r}_{2} \neq 0$, but also $\mathbf{n}_{1}=0$, with $\mathbf{n}_{2} \neq 0$. Then at such a point

$$
\begin{array}{rll}
\mathbf{n} \cdot \mathbf{r}_{2}=0 & \text { and } & \mathbf{n}_{2} \cdot \mathbf{r}=0 \\
\mathbf{n} \cdot \mathbf{r}_{3}=0 & \text { and } & \mathbf{n}_{3} \cdot \mathbf{r}=0 \\
\mathbf{n} \cdot \mathbf{r}_{4}+3 \mathbf{n}_{2} \cdot \mathbf{r}_{2}=0 & \text { and } & 3 \mathbf{n}_{2} \cdot \mathbf{r}_{2}+\mathbf{n}_{4} \cdot \mathbf{r}=0 .
\end{array}
$$

Now $\mathbf{r}, \mathbf{n}$ and $\mathbf{r}_{2}$ form a basis for $\mathbf{R}^{3}$, while $\mathbf{n}_{2} \cdot \mathbf{r}=0$ and $\mathbf{n}_{2} \cdot \mathbf{n}=0$, so, since $\mathbf{n}_{2} \neq 0$, $\mathbf{n}_{2} \cdot \mathbf{r}_{2} \neq 0$, implying that also $\mathbf{n} \cdot \mathbf{r}_{4} \neq 0$ and $\mathbf{n}_{4} \cdot \mathbf{r} \neq 0$. 
Proposition 2. At a point of a curve $\mathbf{r}$ on $S^{2}$, with dual $\mathbf{n}$, where $\mathbf{r}_{1}=0$, with $\mathbf{r}_{2} \neq 0$, and also $\mathbf{n}_{1}=0$, with $\mathbf{n}_{2} \neq 0$, then $\mathbf{r}$ has a rhamphoid cusp. Let $\mathbf{r}_{3}=3 \lambda \mathbf{r}_{2}$ and $\mathbf{n}_{3}=3 \mu \mathbf{n}_{2}$. Then the cusp is ordinary if $\lambda \neq \mu$, special if $\lambda=\mu$. Moreover the dual $\mathbf{n}$ has a rhamphoid cusp of the same type as $\mathbf{r}$.

Proof. From $\mathbf{r} \cdot \mathbf{r}_{2}=0$ and $\mathbf{r} \cdot \mathbf{r}_{3}=0$ it follows that $\mathbf{r}_{3}$ is a multiple of $\mathbf{r}_{2}$, say $\mathbf{r}_{3}=3 \lambda \mathbf{r}_{2}$. Likewise from $\mathbf{n} \cdot \mathbf{r}_{2}=0$ and $\mathbf{n} \cdot \mathbf{r}_{3}=0$ it follows that $\mathbf{n}_{3}$ is a multiple of $\mathbf{n}_{2}$, say $\mathbf{n}_{3}=3 \mu \mathbf{n}_{2}$. From $\mathbf{n} \cdot \mathbf{r}_{4} \neq 0$ it follows that $\mathbf{r}_{4}$ is not a multiple of $\mathbf{r}_{2}$. So $\mathbf{r}$ has a rhamphoid cusp. Likewise $\mathbf{n}$ has a rhamphoid cusp.

As to their type, a further differentiation yields the equation

$$
\mathbf{n} \cdot \mathbf{r}_{5}+4 \mathbf{n}_{1} \cdot \mathbf{r}_{4}+6 \mathbf{n}_{2} \cdot \mathbf{r}_{3}+4 \mathbf{n}_{3} \cdot \mathbf{r}_{2}+\mathbf{n}_{4} \cdot \mathbf{r}_{1}=0
$$

With $\mathbf{n}_{1}=0$ and $\mathbf{r}_{1}=0$, and with $\mathbf{r}_{3}=3 \lambda \mathbf{r}_{2}$ and $\mathbf{n} \cdot \mathbf{r}_{4}+3 \mathbf{n}_{2} \cdot \mathbf{r}_{2}=0$,

$$
\begin{aligned}
\mathbf{n} \cdot \mathbf{r}_{5}-10 \lambda \mathbf{n} \cdot \mathbf{r}_{4} & =-6 \mathbf{n}_{2} \cdot \mathbf{r}_{3}-4 \mathbf{n}_{3} \cdot \mathbf{r}_{2}+3 \mathbf{n}_{2} \cdot \mathbf{r}_{2}=(-18 \lambda-12 \mu+30 \lambda) \mathbf{n}_{2} \cdot \mathbf{r}_{2} \\
& =12(\lambda-\mu) \mathbf{n}_{2} \cdot \mathbf{r}_{2}=0 \quad \text { if and only if } \mu=\lambda .
\end{aligned}
$$

But then $\mathbf{r}_{5}-10 \lambda \mathbf{r}_{4}=\alpha \mathbf{r}+\beta \mathbf{r}_{2}$, for some $\alpha$ and $\beta$, with

$$
\alpha=\mathbf{r} \cdot \mathbf{r}_{5}-10 \lambda \mathbf{r} \cdot \mathbf{r}_{4}=-10 \mathbf{r}_{2} \cdot \mathbf{r}_{3}+30 \lambda \mathbf{r}_{2} \cdot \mathbf{r}_{2}=0 .
$$

That is the rhamphoid cusp on $\mathbf{r}$ is special if and only if $\mathbf{n}_{3}=3 \lambda \mathbf{n}_{2}$. An analogous discussion then proves that the latter equation holds if and only if $\mathbf{n}_{5}=\alpha^{\prime} \mathbf{n}_{2}+10 \lambda \mathbf{n}_{4}$, for some $\alpha^{\prime}$. That is the rhamphoid cusp on the dual curve $\mathbf{n}$ is ordinary or special according as the rhamphoid cusp on $\mathbf{r}$ is ordinary or special.

Note that by this proof we have an alternative characterisation of ordinary or special rhamphoid cusps, namely that if $\mathbf{r}$ has a rhamphoid cusp with $\mathbf{r}_{3}=3 \lambda \mathbf{r}_{2}$, then the cusp is ordinary or special according as $\mathbf{n}_{3} \neq 3 \lambda \mathbf{n}_{2}$ or $\mathbf{n}_{3}=3 \lambda \mathbf{n}_{2}$.

All this is for a curve $\mathbf{r}$ on $S^{2}$. We leave it to the reader to re-examine rhamphoid cusps of plane curves to see whether this fresh characterisation of rhamphoid cusps is valid there, or not. (It is!)

As outlined in Porteous [2], Chapter 5, the theory of parallels and evolutes or caustics of plane curves extends in obvious ways to curves on $S^{2}$. It is immediate that all the parallels to $\mathbf{r}$, including the dual $\mathbf{n}$, have the same derivative curve $\mathbf{t}$. The equations for the evolute e of a regular curve $\mathbf{r}$ on $S^{2}$ are simpler than those for a plane curve, since circles on $S^{2}$ are cut out by planes in $\mathbf{R}^{3}$. The equations defining $\mathbf{e}$ are in fact $\mathbf{e} \cdot \mathbf{e}=1$, $\mathbf{e} \cdot \mathbf{r}_{1}=0$ and $\mathbf{e} \cdot \mathbf{r}_{2}=0$, these defining $\mathbf{e}$ uniquely, if the co-orientation of $\mathbf{r}$ is taken into account. It is then obvious that the evolute or caustic $\mathbf{e}$ is dual to the derivative curve $\mathbf{t}$.

As we have already remarked, the evolute e of a regular curve $\mathbf{r}$ has no geodesic inflections. The analogue of the de l'Hôpital theorem is the following:

Proposition 3. Where a curve $\mathbf{r}$ on $S^{2}$ has an ordinary rhamphoid cusp the evolute e has an ordinary geodesic inflection, and conversely.

Proof. Since $\mathbf{e} \cdot \mathbf{r}_{1}=0$ and $\mathbf{e} \cdot \mathbf{r}_{2}=0$ one also has $\mathbf{e}_{1} \cdot \mathbf{r}_{1}=0$ and therefore

$$
\begin{aligned}
& \mathbf{e} \cdot \mathbf{r}_{3}+\mathbf{e}_{1} \cdot \mathbf{r}_{2}=0 \text { and } \mathbf{e}_{1} \cdot \mathbf{r}_{2}+\mathbf{e}_{2} \cdot \mathbf{r}_{1}=0 \text {, } \\
& \mathbf{e} \cdot \mathbf{r}_{4}+2 \mathbf{e}_{1} \cdot \mathbf{r}_{3}+\mathbf{e}_{2} \cdot \mathbf{r}_{2}=0 \text { and } \mathbf{e}_{1} \cdot \mathbf{r}_{3}+2 \mathbf{e}_{2} \cdot \mathbf{r}_{2}+\mathbf{e}_{3} \cdot \mathbf{r}_{1}=0 \text {, } \\
& \mathbf{e} \cdot \mathbf{r}_{5}+3 \mathbf{e}_{1} \cdot \mathbf{r}_{4}+3 \mathbf{e}_{2} \cdot \mathbf{r}_{3}+\mathbf{e}_{3} \cdot \mathbf{r}_{2}=0 \quad \text { and } \quad \mathbf{e}_{1} \cdot \mathbf{r}_{4}+3 \mathbf{r}_{2} \cdot \mathbf{r}_{3}+3 \mathbf{e}_{3} \cdot \mathbf{r}_{2}+\mathbf{e}_{2} \cdot \mathbf{r}_{1}=0
\end{aligned}
$$


All the terms in the first three of these equations are zero, as is $3 \mathbf{e}_{2} \cdot \mathbf{r}_{3}$, and if any of the others is non-zero so are the others. With $\mathbf{e}, \mathbf{r}_{2}$ and $\mathbf{r}_{4}$ a basis of $\mathbf{R}^{3}$ at a rhamphoid cusp then $\mathbf{e} \cdot \mathbf{r}_{4} \neq 0$ if and only if $\mathbf{e}_{1} \neq 0$. This is indeed the case if and only if the rhamphoid cusp on $\mathbf{r}$ is ordinary, and then $\mathbf{e} \cdot \mathbf{r}_{2}=0, \mathbf{e}_{1} \cdot \mathbf{r}_{2}=0$ and $\mathbf{e}_{2} \cdot \mathbf{r}_{2}=0$, but $\mathbf{e}_{3} \cdot \mathbf{r}_{2} \neq 0$; that is $\mathbf{e}$ then has an ordinary geodesic inflection.

For a regular curve $\mathbf{r}$ on $S^{2}$ the appropriate notion of curvature is the geodesic curvature $\kappa=\cot \theta$, where $\theta$ is the angle between the vectors $\mathbf{r}$ and $\mathbf{e}$, this measuring the extent to which the curve departs from being a great circle. Then $\kappa \mathbf{r}_{1}+\mathbf{n}_{1}=0$, from which it follows that $\kappa_{1} \mathbf{r}_{1}+\kappa \mathbf{r}_{2}+\mathbf{n}_{2}=0$ and $\kappa_{2} \mathbf{r}_{1}+2 \kappa_{1} \mathbf{r}_{2}+\kappa \mathbf{r}_{3}+\mathbf{n}_{3}=0$.

Proposition 4. A rhamphoid cusp on a curve $\mathbf{r}$ on $S^{2}$ is ordinary if and only if the geodesic curvature is not critical there.

Proof. At a rhamphoid cusp $\mathbf{r}_{1}=0$, with $\mathbf{r}_{2} \neq 0, \mathbf{r}_{3}=3 \lambda \mathbf{r}_{2}$ and $\mathbf{n}_{3}=\mu \mathbf{n}_{3}$, the cusp being ordinary if and only if $\mu \neq \lambda$. However, with $\mathbf{r}_{1}=0$ we have $\kappa \mathbf{r}_{2}+\mathbf{n}_{2}=0$ and $2 \kappa_{1} \mathbf{r}_{2}+\kappa \mathbf{r}_{3}+\mathbf{n}_{3}=0$, from which it follows at once that $\kappa_{1} \neq 0$ if and only if $\mu \neq \lambda$.

3. Surfaces in $S^{3}$. Let $\mathbf{s}$ be an oriented and co-oriented regular parametric surface in $S^{3}$, the unit sphere in $\mathbf{R}^{4}$, with parameter space an open subset of $\mathbf{R}^{4}$. The word 'co-oriented' implies that a choice of unit normal $\mathbf{n}$ is made smoothly at each point. We define the surface $\mathbf{n}$ to be the dual surface to $\mathbf{s}$. It is parallel to $\mathbf{r}$ at angular distance $\frac{\pi}{2}$. One has not only $\mathbf{s} \cdot \mathbf{s}=1$ and $\mathbf{n} \cdot \mathbf{n}=1$ but also $\mathbf{n} \cdot \mathbf{s}=0$ and $\mathbf{n} \cdot \mathbf{s}_{1}=0$, from which it follows at once that also $\mathbf{n}_{1} \cdot \mathbf{s}=0$. The dual of the dual is then the surface $-\mathbf{s}$ antipodal to the surface $\mathbf{s}$.

The dual $\mathbf{n}$ of a co-oriented singular surface $\mathbf{s}$ in $S^{3}$ is likewise defined. Not all singular surfaces are co-orientable. The standard example of a surface which is not is the Whitney umbrella.

For any vector $\mathbf{u} \in \mathbf{R}^{2}$ the vector $\mathbf{s}_{1} \mathbf{u}$ is tangent to $\mathbf{s}$ and the vector $\mathbf{n}_{1} \mathbf{u}$ is tangent to $\mathbf{n}$. Suppose first that $\mathbf{s}$ is regular, that is $\mathbf{s}_{1} \mathbf{u} \neq 0$ whenever $\mathbf{u} \neq 0$. Then, although each is orthogonal to $\mathbf{n}, \mathbf{n}_{1} \mathbf{u}$ in general is not a multiple of $\mathbf{s}_{1} \mathbf{u}$.

Proposition 5. With $\mathbf{s}$ and $\mathbf{n}$ as above, one has $\mathbf{n}_{1} \mathbf{u}=\kappa \mathbf{s}_{1} \mathbf{u}$, for some $\kappa$, and some non-zero vector $\mathbf{u}$, if and only if $\mathbf{n} \cdot \mathbf{s}_{2} \mathbf{u}=\kappa \mathbf{s}_{1} \cdot \mathbf{s}_{1} \mathbf{u}$, there being, in general, distinct real $\kappa, \lambda$ and distinct real non-zero $\mathbf{u}, \mathbf{v}$ such that

$$
\mathbf{n} \cdot \mathbf{s}_{2} \mathbf{u}=\kappa \mathbf{s}_{1} \cdot \mathbf{s}_{1} \mathbf{u} \quad \text { and } \quad \mathbf{n} \cdot \mathbf{s}_{2} \mathbf{v}=\lambda \mathbf{s}_{1} \cdot \mathbf{s}_{1} \mathbf{v},
$$

with $\mathbf{n} \cdot \mathbf{s}_{2} \mathbf{u v}=0$ and $\mathbf{s}_{1} \mathbf{u} \cdot \mathbf{s}_{1} \mathbf{v}=0$.

Proof. Standard eigenvalue problem.

The eigenvalues $\kappa$ and $\lambda$ are then defined to be the principal (geodesic) curvatures of $\mathbf{s}$ and the vectors $\mathbf{s}_{1} \mathbf{u}$ and $\mathbf{s}_{1} \mathbf{v}$ the principal tangent vectors of $\mathbf{s}$, the latter being mutually orthogonal provided that $\kappa$ and $\lambda$ are not equal. We normalise these to be of unit length, setting $\mathbf{u}=\mathbf{a}_{1}$ and $\mathbf{v}=\mathbf{b}_{1}$, these vectors being unique up to sign. By analogy with the theory of surfaces in $\mathbf{R}^{3}$ the symmetric twice-linear forms $\mathbf{s}_{1} \cdot \mathbf{s}_{1}$ and $\mathbf{n} \cdot \mathbf{s}_{2}$ are called the first and second fundamental forms of the surface $\mathbf{s}$. Note that since $\mathbf{n} \cdot \mathbf{s}_{1}=0$ we have $\mathbf{n}_{1} \cdot \mathbf{s}_{1}=-\mathbf{n} \cdot \mathbf{s}_{2}$ a symmetric twice-linear form. 
The principal tangent vectors are tangent to two mutually orthogonal foliations of the surface by lines of curvature, just as for surfaces in $\mathbf{R}^{3}$.

The parabolic line on a regular surface $\mathbf{s}$ on $S^{3}$ consists of those points where the twice-linear form $\mathbf{n} \cdot \mathbf{s}_{2}$ is parabolic, that is where there is a non-zero vector $\mathbf{u}$ in $\mathbf{R}^{2}$ such that $\mathbf{n} \cdot \mathbf{s}_{2} \mathbf{u}=0$, an equivalent condition being that $\mathbf{n}_{1} \mathbf{u}=0$; for $\mathbf{s}, \mathbf{n}$ and the image of $\mathbf{s}_{1}$ span $\mathbf{R}^{4}$ and $\mathbf{s} \cdot \mathbf{n}_{1}=0, \mathbf{n} \cdot \mathbf{n}_{1}=0$, while $\mathbf{s}_{1} \cdot \mathbf{n}_{1}=-\mathbf{n} \cdot \mathbf{s}_{2}$. For a co-oriented singular surface these conditions are no longer equivalent. It seems that the appropriate definition in that case is to say that the parabolic line consists of those points where there is a non-zero vector $\mathbf{u}$ in $\mathbf{R}^{2}$ such that $\mathbf{n}_{1} \mathbf{u}=0$.

A co-oriented surface $\mathbf{s}$ is said to have a cuspidal edge where there is a non-zero vector $\mathbf{u}$ such that $\mathbf{s}_{1} \mathbf{u}=0$. With this definition it is immediate that where a co-oriented surface has a parabolic line the dual surface has a cuspidal edge.

Anyone familiar with the classical result of Whitney will expect it to be the case that a map $\mathbf{s}: \mathbf{R}^{2} \longrightarrow S^{3}\left(^{1}\right)$ in general only fails to be regular at isolated points, these being Whitney umbrellas. That this is not the case here is due to the fact that we are restricting to co-orientable parametrised surfaces, the image of the first differential of $\mathbf{s}$ at any point lying in the orthogonal complement of the unit normal vector $\mathbf{n}$.

With $\mathbf{s}$ and $\mathbf{n}$ as above we say that $\mathbf{s}$ has an ordinary parabolic line and $\mathbf{n}$ an ordinary cuspidal edge where not only the dimension of the kernel of $\mathbf{n}_{1}$ is one, with $\mathbf{n}_{1} \mathbf{u}=0$, for some non-zero vector $\mathbf{u}$, but also $\mathbf{n}_{2} \mathbf{u}^{2}+\mathbf{n}_{1} \mathbf{v} \neq 0$ for all $\mathbf{v} \in \mathbf{R}^{2}$.

Proposition 6. With $\mathbf{s}$ and $\mathbf{n}$ as above, the points of the ordinary parabolic line form a one-dimensional submanifold of $S^{3}$ lying in the image of $\mathbf{s}$, while the points of the dual cuspidal edge form a one-dimensional submanifold of $S^{3}$ lying in the image of $\mathbf{n}$, provided that the intersection of the kernels of $\mathbf{s}_{1}$ and $\mathbf{n}_{1}$ is everywhere zero.

Proof. At each point of the parabolic line of $\mathbf{s}: \mathbf{c} \mapsto \mathbf{s}(\mathbf{c})$ or cuspidal edge of $\mathbf{n}: \mathbf{c} \mapsto \mathbf{n}(\mathbf{c})$ there is a non-zero vector $\mathbf{u}$ such that $\mathbf{n}_{1} \mathbf{u}=0$. By the rank theorem it is enough to prove that the rank of the map

$$
\begin{gathered}
\mathbf{R}^{2} \times \mathbf{R}^{2}>\mathbf{R}^{4} \\
(\mathbf{c}, \mathbf{u}) \mapsto \mathbf{n}_{1} \mathbf{u}
\end{gathered}
$$

is everywhere equal to 2 , where the argument $\mathbf{c}$ is omitted on the right-hand side.

Let $\left(\mathbf{c}_{1}, \mathbf{u}_{1}\right)$ be a kernel vector. Then

$$
\mathbf{n}_{2} \mathbf{c}_{1} \mathbf{u}+\mathbf{n}_{1} \mathbf{u}_{1}=0
$$

implying that $\mathbf{s}_{1} \mathbf{u} \cdot \mathbf{n}_{2} \mathbf{u c}_{1}=0$.

Now it follows from the fact that $\mathbf{s}_{1} \mathbf{u} \cdot \mathbf{n}_{2} \mathbf{u}^{2} \neq 0$ that $\mathbf{s}_{1} \mathbf{u} \cdot \mathbf{n}_{2} \mathbf{u} \neq 0$, with a non-zero kernel vector $\mathbf{c}_{1}$ not a multiple of $\mathbf{u}$. For it is not hard to verify that if $\mathbf{s}_{1} \mathbf{u} \cdot \mathbf{n}_{2} \mathbf{u}^{2}=0$ then there exists a non-zero vector $\mathbf{v}$ such that $\mathbf{n}_{2} \mathbf{u}^{2}+\mathbf{n}_{1} \mathbf{v}=0$, contrary to hypothesis.

With the dimension of the kernel of $\mathbf{n}_{1}$ equal to 1 the vector $\mathbf{u}_{1}$ is then determined modulo $\mathbf{u}$.

One then proceeds to analyse what happens when the condition of ordinariness fails. In the case of a surface in $\mathbf{R}^{3}$ this leads to the study of 'cusps of Gauss'. See Porteous [2],

$\left({ }^{1}\right)$ The tail on the arrow indicates that the domain is an open subset of the source indicated. 
pages 217-221. In the present situation it turns out that at the analogue of a cusp of Gauss on the parabolic line of $\mathbf{s}$ the parabolic line is simply tangential to the associated line of curvature, while the dual has a swallow-tail.

Proposition 7. With the notations of the previous proposition, suppose still that, at some point $\mathbf{p}, \mathbf{s}_{1} \mathbf{u} \cdot \mathbf{n}_{2} \mathbf{u} \neq 0$, but that $\mathbf{s}_{1} \mathbf{u} \cdot \mathbf{n}_{2} \mathbf{u}^{2}=0$, or equivalently $\mathbf{n}_{2} \mathbf{u}^{2}+\mathbf{n}_{1} \mathbf{v}=0$, for some vector $\mathbf{v}$, while there is no vector $\mathbf{w}$ such that $\mathbf{n}_{3} \mathbf{u}^{3}+3 \mathbf{n}_{2} \mathbf{u v}+\mathbf{n}_{1} \mathbf{w}=0$. Then at $\mathbf{p}$ the parabolic line on $\mathbf{s}$ remains a one-dimensional submanifold of the image of $\mathbf{s}$, but with tangent line coinciding with the tangent line to the associated line of curvature there, the contact with that line being simple, while the cuspidal edge on $\mathbf{n}$ has an ordinary cusp, this point being a swallow-tail point on $\mathbf{n}$.

Pr o of. That $\mathbf{c}_{1}$ is a multiple of $\mathbf{u}$ at $\mathbf{p}$ follows at once from the proof of the previous proposition. That such points are isolated follows from verifying that the only kernel vectors of the map

$$
\begin{gathered}
\mathbf{R}^{2} \times \mathbf{R}^{2} \times \mathbf{R}^{2}>\mathbf{R}^{4} \times \mathbf{R}^{4} \\
(\mathbf{c}, \mathbf{u}, \mathbf{v}) \mapsto\left(\mathbf{n}_{1} \mathbf{u}, \mathbf{n}_{2} \mathbf{u}^{2}+\mathbf{n}_{1} \mathbf{v}\right)
\end{gathered}
$$

are of the form $\left(0, \mathbf{u}_{1}, \mathbf{v}_{1}\right)$ where $\mathbf{u}_{1}$ and $\mathbf{v}_{1}$ are uniquely determined $\bmod \mathbf{u}$. In fact one must have

$$
\mathbf{n}_{2} \mathbf{c}_{1} \mathbf{u}+\mathbf{n}_{1} \mathbf{u}_{1}=0
$$

implying as before that

$$
\mathbf{s}_{1} \mathbf{u} \cdot \mathbf{n}_{2} \mathbf{u} \mathbf{c}_{1}=0
$$

and therefore that $c_{1}=\lambda \mathbf{u}$ and $\mathbf{u}_{1}=\lambda \mathbf{v} \bmod \mathbf{u}$, and

$$
\mathbf{n}_{3} \mathbf{c}_{1} \mathbf{u}^{2}+2 \mathbf{n}_{2} \mathbf{u} \mathbf{u}_{1}+\mathbf{n}_{2} c_{1} \mathbf{v}+\mathbf{n}_{1} \mathbf{v}_{1}=0
$$

that is

$$
\lambda\left(\mathbf{n}_{3} \mathbf{u}^{3}+3 \mathbf{n}_{2} \mathbf{u v}+\mathbf{n}_{1} \mathbf{v}\right)=0
$$

implying that $\mathbf{s}_{1} \mathbf{u} \cdot \lambda\left(\mathbf{n}_{3} \mathbf{u}^{3}+3 \mathbf{n}_{2} \mathbf{u v}\right)=0$, and therefore that $\lambda\left(\mathbf{n}_{3} \mathbf{u}^{3}+3 \mathbf{n}_{2} \mathbf{u v}\right)+\mathbf{n}_{1} \mathbf{w}=0$, for some vector $\mathbf{w}$. But this can only be the case if $\lambda=0$.

Now let $\mathbf{c}$ denote the representative curve in the parameter space of the parabolic line and the cuspidal edge, with $\mathbf{c}_{1}=\mathbf{u} \neq 0$ at $\mathbf{p}$. Then the successive derivatives of the parabolic line $\mathbf{s c}$ are $(\mathbf{s c})_{1}=\mathbf{s}_{1} \mathbf{c}_{1} \neq 0$, equal to $\mathbf{s}_{1} \mathbf{u}$ at $\mathbf{p}$, and $(\mathbf{s c})_{2}=\mathbf{s}_{2} \mathbf{c}_{1}^{2}+\mathbf{s}_{1} \mathbf{c}_{2}$, while those of the cuspidal edge $\mathbf{n c}$ are $(\mathbf{n c})_{1}=\mathbf{n}_{1} \mathbf{c}_{1} \neq 0$ except at a point such as $\mathbf{p}$, where it is $0,(\mathbf{n c})_{2}=\mathbf{n}_{2} \mathbf{c}_{1}^{2}+\mathbf{n}_{1} \mathbf{c}_{2}$ and $(\mathbf{n c})_{3}$. Now differentiating the equation $\mathbf{n}_{1} \mathbf{u}=0$ along $\mathbf{c}$ we have

reducing at $\mathbf{p}$ to

$$
\mathbf{n}_{2} \mathbf{u c} \mathbf{c}_{1}+\mathbf{n}_{1} \mathbf{u}_{1}=0
$$

$$
\mathbf{n}_{2} \mathbf{u}^{2}+\mathbf{n}_{1} \mathbf{v}=0
$$

with $\mathbf{u}_{1}=\mathbf{v} \bmod \mathbf{u}$ and

$$
\mathbf{n}_{2} \mathbf{u c}_{2}+\mathbf{n}_{3} \mathbf{u} \mathbf{c}_{1}^{2}+2 \mathbf{n}_{2} \mathbf{c}_{1} \mathbf{u}_{1}+\mathbf{n}_{1} \mathbf{u}_{2}=0
$$

reducing at $\mathbf{p}$ to

$$
\mathbf{n}_{2} \mathbf{u} \mathbf{c}_{2}+\mathbf{n}_{3} \mathbf{u}^{3}+2 \mathbf{n}_{2} \mathbf{u v}+\mathbf{n}_{1} \mathbf{u}_{2}=0
$$


Now were we to have $\mathbf{c}_{2}=\mathbf{v} \bmod \mathbf{u}$ we would immediately have a contradiction, on forming the dot product with $\mathbf{s}_{1} \mathbf{u}$ and recalling that there is no vector $\mathbf{w}$ such that $\mathbf{n}_{3} \mathbf{u}^{3}+3 \mathbf{n}_{2} \mathbf{u v}+\mathbf{n}_{1} \mathbf{w}=0$.

It follows that at $\mathbf{p}$ the vector $(\mathbf{s c})_{2}$ is not the second derivative of the line of curvature to which the parabolic line is tangent, that is the contact between the two lines is simple, while $(\mathbf{n c})_{1}=0$, though $(\mathbf{n c})_{2} \neq 0$.

It remains to prove that $(\mathbf{n c})_{3}$ is not a multiple of $(\mathbf{n c})_{2}$. We remark first of all that at $\mathbf{p}$ the vectors $\mathbf{s} . \mathbf{n}, \mathbf{s}_{1} \mathbf{u}$ and $\left(\mathbf{s}_{1} \mathbf{u}\right)_{1} \mathbf{u}$, form a basis for $\mathbf{R}^{4}$. For differentiate the equation

$$
\mathbf{n}_{1} \cdot \mathbf{s}_{1} \mathbf{u}=0
$$

along c. Then we get

$$
\mathbf{n}_{1} \cdot\left(\mathbf{s}_{1} \mathbf{u}\right)_{1}+\mathbf{n}_{2} \mathbf{c}_{1} \cdot \mathbf{s}_{1} \mathbf{u}=0
$$

with $\mathbf{c}_{1}$ a multiple of $\mathbf{u}$. But $\mathbf{s}_{1} \mathbf{u} \cdot \mathbf{n}_{2} \mathbf{u} \neq 0$, from which it follows that $\mathbf{n}_{1} \cdot\left(\mathbf{s}_{1} \mathbf{u}\right)_{1} \neq 0$. The assertion follows at once. (Remark: this means that the associated line of curvature through $\mathbf{p}$, with $\mathbf{u}$ as tangent vector, does not have a geodesic inflection at $\mathbf{p}$.)

Now differentiate the equation

$$
\mathbf{s}_{1} \mathbf{u} \cdot(\mathbf{n c})_{1}=\mathbf{s}_{1} \mathbf{c}_{1} \cdot \mathbf{n}_{1} \mathbf{u}=0
$$

twice along $\mathbf{c}$ to get

$$
\mathbf{s}_{1} \mathbf{u} \cdot(\mathbf{n c})_{2}+\left(\mathbf{s}_{1} \mathbf{u}\right)_{1} \cdot(\mathbf{n c})_{1}
$$

reducing at $\mathbf{p}$ to

$$
\mathbf{s}_{1} \mathbf{u} \cdot(\mathbf{n c})_{2}=0
$$

and

$$
\mathbf{s}_{1} \mathbf{u} \cdot(\mathbf{n c})_{3}+2\left(\mathbf{s}_{1} \mathbf{u}\right)_{1} \cdot(\mathbf{n c})_{2}+\left(\mathbf{s}_{1} \mathbf{u}\right)_{2} \cdot(\mathbf{n c})_{1}=0
$$

reducing at $\mathbf{p}$ to

$$
\mathbf{s}_{1} \mathbf{u} \cdot(\mathbf{n c})_{3}+2\left(\mathbf{s}_{1} \mathbf{u}\right)_{1} \cdot(\mathbf{n c})_{2}=0 .
$$

From the fact that $(\mathbf{n c})_{2} \neq 0$ it follows that, at $\mathbf{p},\left(\mathbf{s}_{1} \mathbf{u}\right)_{1} \cdot(\mathbf{n c})_{2} \neq 0$ and hence that $\mathbf{s}_{1} \mathbf{u} \cdot(\mathbf{n c})_{3} \neq 0$. That is $(\mathbf{n c})_{3}$ is not a multiple of $(\mathbf{n c})_{2}$ at $\mathbf{p}$, which is what had to be proved.

One may go on to show that to a birth-point or normal crossing of the parabolic line there corresponds a lips birth-point of the cuspidal edge or a beak-to-beak singularity of the cuspidal edge. We leave this to the reader to verify.

It should be clear from our definitions of parabolic line and cuspidal edge that such lines on a surface may cross each other transversally, it being in general the case that either locally separates parts of the surface of positive Gaussian curvature from parts of negative Gaussian curvature, the Gaussian curvature being defined to be the product of the principal curvatures, just as for surfaces in $\mathbf{R}^{3}$. What is more remarkable is that there is nothing in the definition to prevent a line being at the same time an ordinary parabolic line and an ordinary cuspidal edge. This will not be a rhamphoid cuspidal edge, as one might at first conjecture by analogy with the situation noted earlier for curves in $S^{2}$, provided that the kernels of the differentials of $\mathbf{s}$ and $\mathbf{n}$ have zero intersection. Such lines arise naturally when reflectional symmetry is present, as we shall shortly find. On either side of such a line the Gaussian curvature will have the same sign! 
Since 2-spheres on $S^{3}$ are cut out by hyperplanes in $\mathbf{R}^{4}$ the theory of the evolute is simpler than for a surface in $\mathbf{R}^{3}$. With $\mathbf{s}$, a basis for the image of $\mathbf{s}_{1}$ and $\mathbf{n}$ forming a basis for $\mathbf{R}^{4}$ at each point of the regular surface $\mathbf{s}$ it follows that where $\mathbf{e} \cdot \mathbf{e}=1$ and $\mathbf{e} \cdot \mathbf{s}_{1}=0$ then $\mathbf{e}=\mathbf{s} \cos \theta+\mathbf{n} \sin \theta$. Then

$$
\mathbf{e} \cdot \mathbf{s}_{2}=\mathbf{s} \cdot \mathbf{s}_{2} \cos \theta+\mathbf{n} \cdot \mathbf{s}_{2} \sin \theta=\left(\mathbf{n} \cdot \mathbf{s}_{2}-\mathbf{s}_{1} \cdot \mathbf{s}_{1} \cot \theta\right) \sin \theta .
$$

We have already solved the resulting eigenvalue problem, yielding at each point real eigenvalues $\kappa=\cot \theta$ and $\lambda=\cot \phi$ and non-zero real eigenvectors $\mathbf{u}$ and $\mathbf{v}$ such that

$$
\mathbf{n} \cdot \mathbf{s}_{2} \mathbf{u}=\kappa \mathbf{s}_{1} \cdot \mathbf{s}_{1} \mathbf{u} \text { and } \mathbf{n} \cdot \mathbf{s}_{2} \mathbf{v}=\lambda \mathbf{s}_{1} \cdot \mathbf{s}_{1} \mathbf{v} .
$$

The eigenvalues $\kappa$ and $\lambda$ are just the principal (geodesic) curvatures of $\mathbf{s}$ and the vectors $\mathbf{s}_{1} \mathbf{u}$ and $\mathbf{s}_{1} \mathbf{v}$ the principal tangent vectors of $\mathbf{s}$, the latter being mutually orthogonal provided that $\kappa$ and $\lambda$ are not equal. We normalise these to be of unit length, setting $\mathbf{u}=\mathbf{a}_{1}$ and $\mathbf{v}=\mathbf{b}_{1}$, these vectors being unique up to sign. Then there are two sheets $\mathbf{e}$ and $\mathbf{f}$ of the evolute or caustic of $\mathbf{s}$ defined by $\mathbf{e} \cdot \mathbf{e}=1$, with $\mathbf{e} \cdot \mathbf{s}_{1}=0$ and $\mathbf{e} \cdot \mathbf{s}_{2} \mathbf{a}_{1}=0$, and $\mathbf{f} \cdot \mathbf{f}=1$, with $\mathbf{f} \cdot \mathbf{s}_{1}=0$ and $\mathbf{f} \cdot \mathbf{s}_{2} \mathbf{b}_{1}=0$.

A co-oriented regular surface on $S^{3}$ is said to have an umbilic where the principal curvatures $\kappa$ and $\lambda$ are equal. Ignoring such points for the time being we have:

Proposition 8. Let $\mathbf{s}$ be a co-oriented surface in $S^{3}$. Then, with the above notations, $\mathbf{s}_{1} \mathbf{a}_{1}$ is the unit normal to $\mathbf{e}$, and therefore is dual to $\mathbf{e}$, while $\mathbf{f}_{1} \mathbf{b}_{1}$ is the unit normal to $\mathbf{f}$, and therefore is dual to $\mathbf{f}$.

Proof. Since $\mathbf{e} \cdot \mathbf{s}_{1}=0$ it follows that $\mathbf{e} \cdot \mathbf{s}_{2}+\mathbf{e}_{1} \cdot \mathbf{s}_{1}=0$ and since $\mathbf{e} \cdot \mathbf{s}_{2}$ is symmetric in its two slots, so also is $\mathbf{e}_{1} \cdot \mathbf{s}_{1}$. From $\mathbf{e} \cdot \mathbf{s}_{2} \mathbf{a}_{1}=0$ it then follows that $\mathbf{e}_{1} \cdot \mathbf{s}_{1} \mathbf{a}_{1}=0$, which is what had to be proved. The other sheet is dealt with analogously.

The surfaces $\mathbf{s}_{1} \mathbf{a}_{1}$ and $\mathbf{s}_{1} \mathbf{b}_{1}$ form the two sheets of the derivative of the surface $\mathbf{s}$.

Ridges on a surface are those curves where one or other of the principal curvatures is critical in the associated principal direction, the associated sheet of the evolute having a cuspidal edge. On the other hand, a flexcord, consisting of points where the other principal curvature is critical in a principal direction, and which also consists of points where the other line of curvature has a geodesic inflection, lies under a parabolic line on the evolute, the corresponding curve on the derivative accordingly being a cuspidal edge.

In the special case of a line of reflectional symmetry on the surface this is at the same time both a ridge and a flexcord, of the same 'colour'. On the relevant sheet of the evolute we have a curve that is at the same time an ordinary parabolic line and an ordinary cuspidal edge. For definiteness suppose that the sheet involved is $\mathbf{e}$. Then if $\mathbf{e}_{1}$ has a kernel vector it must be a multiple of $\mathbf{a}_{1}$, while if $\left(\mathbf{s}_{1} \mathbf{a}_{1}\right)_{1}$ has a kernel vector it must be a multiple of $\mathbf{b}_{1}$, so that the kernels of the differentials in this case do not coincide.

What happens at umbilics is another story, which we do not embark upon here!

4. Curves in $S^{3}$. Let $\mathbf{r}$ be a regular curve in $S^{3}$. It is then clear that the unit tangent $\mathbf{t}$ is the derivative of $\mathbf{r}$, but it may not be so clear what one should take as the dual, there being a whole circle of unit vectors normal to the curve at any point. Should one take the tube centred on the curve, with angular radius $\frac{\pi}{2}$, or is some particular normal 
vector to be preferred? A hint to the correct answer is provided by consideration of the evolute or caustic of $\mathbf{r}$. By analogy with what one does for curves in $\mathbf{R}^{3}$ the space evolute $\mathbf{e}$ is defined by the equations $\mathbf{e} \cdot \mathbf{e}=1, \mathbf{e} \cdot \mathbf{r}_{1}=0, \mathbf{e} \cdot \mathbf{r}_{2}=0$ and $\mathbf{e} \cdot \mathbf{r}_{3}=0$, at least where the vectors $\mathbf{r}_{1}, \mathbf{r}_{2}$ and $\mathbf{r}_{3}$ are linearly independent, from which it follows that for tangent vectors to $\mathbf{e}$ of unit length, $\mathbf{e}_{1} \cdot \mathbf{e}_{1}=1, \mathbf{e}_{1} \cdot \mathbf{r}_{1}=0$ and $\mathbf{e}_{1} \cdot \mathbf{r}_{2}=0$. For a unit-speed curve $\mathbf{r}$, with $\mathbf{t}=\mathbf{r}_{1}$ and $\mathbf{e}_{1}=\mathbf{r}_{2}$, this is seen to be the tube of radius $\frac{\pi}{2}$ with core the derivative curve $\mathbf{t}$.

The following proposition clarifies everything. First we define a curve $\mathbf{r}$ in $S^{3}$ to be co-orientable if there exists a curve $\mathbf{b}$ in $S^{3}$ such that $\mathbf{b} \cdot \mathbf{r}=0, \mathbf{b} \cdot \mathbf{r}_{1}=0$ and $\mathbf{b} \cdot \mathbf{r}_{2}=0$. Such a curve $\mathbf{b}$, the binormal curve, having been taken as the co-orientation of $\mathbf{r}$, is then defined to be the dual of the curve $\mathbf{r}$.

Proposition 9. With $\mathbf{r}$ and $\mathbf{b}$ as above, when $\mathbf{b} \cdot \mathbf{r}_{3} \neq 0$ then $\mathbf{r}_{1} \neq 0$ and $\mathbf{b}_{1}=0$, and the tangent great circle bundle of $\mathbf{b}$ coincides with the tube of angular radius $\frac{\pi}{2}$ with core the curve $\mathbf{r}$.

Proof. From $\mathbf{b} \cdot \mathbf{r}=0, \mathbf{b} \cdot \mathbf{r}_{1}=0$ and $\mathbf{b} \cdot \mathbf{r}_{2}=0$ it follows that $\mathbf{b}_{1} \cdot \mathbf{r}=0, \mathbf{b}_{1} \cdot \mathbf{r}_{1}=0$ and $\mathbf{b}_{2} \cdot \mathbf{r}=0$. Also from $\mathbf{b} \cdot \mathbf{r}_{2}=0$ it follows that $\mathbf{b} \cdot \mathbf{r}_{3}+\mathbf{b}_{1} \cdot \mathbf{r}_{2}=0$, while from $\mathbf{b}_{1} \cdot \mathbf{r}_{1}=0$ it follows that $\mathbf{b}_{1} \cdot \mathbf{r}_{2}+\mathbf{b}_{2} \cdot \mathbf{r}_{1}=0$, So $\mathbf{b} \cdot \mathbf{r}_{3} \neq 0$ implies that $\mathbf{b}_{1} \cdot \mathbf{r}_{2} \neq 0$ and $\mathbf{b}_{2} \cdot \mathbf{r}_{1} \neq 0$, and hence that $\mathbf{b}_{1} \neq 0$ and $\mathbf{r}_{1} \neq 0$. Moreover $\mathbf{r}, \mathbf{b}, \mathbf{r}_{1}$ and $\mathbf{b}_{1}$ form an orthogonal basis for $\mathbf{R}^{4}$ at such a point. Choose a parametrisation for the curve such that $\mathbf{b}$ is unit-speed. (Here and elsewhere throughout this paper the various definitions given are all independent of the explicit parametrisation used to define the curve or surface.) Then the points $\mathbf{b} \cos \theta+\mathbf{b}_{1} \sin \theta$ form not only the tangent great circle bundle for $\mathbf{b}$ but also the tube of angular radius $\frac{\pi}{2}$ with core the curve $\mathbf{r}$.

Of course this is also the tangent great circle bundle of $-\mathbf{b}$, antipodal to $\mathbf{b}$.

Clearly the derivative $\mathbf{t}$ of $\mathbf{r}$ is the dual of the space evolute $\mathbf{e}$, or of $-\mathbf{e}$, depending on choices of sign that have been made.

Remark: The tangent great circle bundle of a regular curve $\mathbf{r}$ on $S^{3}$ is parabolic everywhere. Its dual surface collapses to the dual curve $\mathbf{b}$ of $\mathbf{r}$.

At a generic point of a curve $\mathbf{r}$ on $S^{3}$ the four vectors $\mathbf{r}, \mathbf{r}_{1}, \mathbf{r}_{2}$ and $\mathbf{r}_{3}$ will be linearly independent, as will be the four vectors $\mathbf{r}_{1}, \mathbf{r}_{2}, \mathbf{r}_{3}$ and $\mathbf{r}_{4}$. Either may fail at particular points in several different ways. At a regular point of $\mathbf{r}$, where $\mathbf{r}_{1} \neq 0$, though $\mathbf{r} \cdot \mathbf{r}_{1}=0$, one has $\mathbf{r} \cdot \mathbf{r}_{2}=\mathbf{r}_{1} \cdot \mathbf{r}_{1} \neq 0$. So $\mathbf{r}_{1}$ and $\mathbf{r}_{2}$ are linearly independent. However it may be that $\mathbf{r}, \mathbf{r}_{1}$ and $\mathbf{r}_{2}$ are coplanar. At such a point we say that $\mathbf{r}$ has a geodesic inflection, ordinary if the vectors $\mathbf{r}, \mathbf{r}_{1}, \mathbf{r}_{3}$ and $\mathbf{r}_{4}$ are linearly independent. By analogy with the case of curves in $\mathbf{R}^{3}$ we say that a curve $\mathbf{r}$ on $S^{3}$ has a vertex at a point where the vectors $\mathbf{r}_{1}, \mathbf{r}_{2}$ and $\mathbf{r}_{3}$ are linearly independent, but the four vectors $\mathbf{r}_{1}, \mathbf{r}_{2}, \mathbf{r}_{3}$ and $\mathbf{r}_{4}$ are linearly dependent, this being an ordinary vertex if the vectors $\mathbf{r}_{1}, \mathbf{r}_{2}, \mathbf{r}_{3}$ and $\mathbf{r}_{5}$ are linearly independent. Also, again by analogy with the case of curves in $\mathbf{R}^{3}$, at a point where the vectors $\mathbf{r}_{1}, \mathbf{r}_{2}$ and $\mathbf{r}_{3}$ are linearly dependent we say that $\mathbf{r}$ has a point of torsion zero, ordinary if the vectors $\mathbf{r}_{1}, \mathbf{r}_{2}, \mathbf{r}_{4}$ and $\mathbf{r}_{5}$ are linearly independent.

Proposition 10. Suppose that at a point of a co-oriented curve $\mathbf{r}$ on $S^{3}$ the vectors $\mathbf{r}, \mathbf{r}_{1}$ and $\mathbf{r}_{2}$ are linearly independent, but $\mathbf{r}_{3}$ depends on these, while $\mathbf{r}_{4}$ does not. Then 
the dual curve $\mathbf{b}$ has a cusp, where $\mathbf{b}_{1}=0$, and $\mathbf{b}_{2}, \mathbf{b}_{3}$ and $\mathbf{b}_{4}$ are linearly independent, and vice versa.

Pr o of. Similar to the proof of Proposition 1, but carried one stage further.

Proposition 11. Let $\mathbf{r}$ be a co-oriented curve on $S^{3}$ with an ordinary vertex. Then at that point the space evolute $\mathbf{e}$ has a cusp where $\mathbf{e}_{1}=0$, and $\mathbf{e}_{2}, \mathbf{e}_{3}$ and $\mathbf{e}_{4}$ are linearly independent.

Proof. This follows immediately from the previous proposition.

Proposition 12. Suppose that a co-oriented regular curve $\mathbf{r}$ in $S^{3}$ has a geodesic inflection, Then not only $\mathbf{b} \cdot \mathbf{r}=0, \mathbf{b} \cdot \mathbf{r}_{1}=0$ and $\mathbf{b} \cdot \mathbf{r}_{2}=0$ but also $\mathbf{b}_{1} \cdot \mathbf{r}=0, \mathbf{b}_{1} \cdot \mathbf{r}_{1}=0$, $\mathbf{b}_{1} \cdot \mathbf{r}_{2}=0$ and $\mathbf{b} \cdot \mathbf{r}_{3}=0$. Moreover $\mathbf{b} \cdot \mathbf{r}_{4}+2 \mathbf{b}_{1} \cdot \mathbf{r}_{3}=0$, implying that at an ordinary geodesic inflection $\mathbf{b}_{1} \neq 0$.

Proof. From $\mathbf{b} \cdot \mathbf{r}=0, \mathbf{b} \cdot \mathbf{r}_{1}=0$ and $\mathbf{b} \cdot \mathbf{r}_{2}=0$ it follows that $\mathbf{b}_{1} \cdot \mathbf{r}=0, \mathbf{b}_{1} \cdot \mathbf{r}_{1}=0$ and $\mathbf{b}_{2} \cdot \mathbf{r}=0$. Also from $\mathbf{b} \cdot \mathbf{r}_{2}=0$ it follows that $\mathbf{b} \cdot \mathbf{r}_{4}+\mathbf{b}_{1} \cdot \mathbf{r}_{3}=0$. Since $\mathbf{r}, \mathbf{r}_{1}$ and $\mathbf{r}_{2}$ are coplanar and since $\mathbf{b}_{1} \cdot \mathbf{r}=0$ and $b_{1} \cdot \mathbf{r}_{1}=0$ it follows that $\mathbf{b}_{1} \cdot \mathbf{r}_{2}=0$ and therefore that $\mathbf{b} \cdot \mathbf{r}_{3}=0$. From $\mathbf{b}_{1} \cdot \mathbf{r}_{1}=0$ it follows that $\mathbf{b}_{1} \cdot \mathbf{r}_{2}+\mathbf{b}_{2} \cdot \mathbf{r}_{1}=0$, and therefore that $\mathbf{b}_{2} \cdot \mathbf{r}_{1}=0$, so that $\mathbf{b}_{2} \cdot \mathbf{r}_{2}=0$. But $\mathbf{b} \cdot \mathbf{r}_{4}+2 \mathbf{b}_{1} \cdot \mathbf{r}_{3}+\mathbf{b}_{2} \cdot \mathbf{r}_{2}=0$, so $\mathbf{b} \cdot \mathbf{r}_{4}+2 \mathbf{b}_{1} \cdot \mathbf{r}_{3}=0$. At an ordinary geodesic inflection $\mathbf{b} \cdot \mathbf{r}_{4} \neq 0$. So $\mathbf{b}_{1} \cdot \mathbf{r}_{3} \neq 0$ and therefore $\mathbf{b}_{1} \neq 0$ at such a point.

Proposition 13. Suppose that at some point a co-oriented regular curve $\mathbf{r}$ in $S^{3}$ has an ordinary geodesic inflection, Then at the corresponding point of the dual curve $\mathbf{b}$ there is also an ordinary geodesic inflection.

Pr o of. This follows at once from the detail of the proof of the previous proposition, for, since $\mathbf{b}_{2} \cdot \mathbf{r}_{2} \neq 0$, the fact that $\mathbf{b}_{1} \cdot \mathbf{r}_{1} \neq 0$ implies that $\mathbf{b}_{3} \cdot \mathbf{r}_{1} \neq 0$ and $\mathbf{b}_{4} \cdot \mathbf{r} \neq 0$.

Remark: The tangent great circle bundle of a regular curve $\mathbf{r}$ on $S^{3}$ has an ordinary cuspidal edge not only along $\mathbf{r}$ but also along the tangent great circle at any ordinary geodesic inflection. At such an inflection the two cuspidal edges are tangent to each other. We omit the details.

We conclude with a brief discussion of the case that $\mathbf{r}$ has torsion zero.

Proposition 14. Let $\mathbf{r}$ be a curve on $S^{3}$ with an ordinary point of torsion zero. Then at that point the derivative $\mathbf{t}$ and the space evolute $\mathbf{e}$ each has an ordinary geodesic inflection.

Pr o of. This follows directly from the definition of 'ordinary' and the previous proposition.

\section{References}

[1] V. I. Arnol'd, The geometry of spherical curves and the algebra of quaternions (in Russian), Uspekhi Mat. Nauk 50:1 (1995), 3-68; Engl. transl.: Russian Math. Surveys 50:1 (1995), 1-68.

[2] I. R. Porteous, Geometric Differentiation for the Intelligence of Curves and Surfaces, Cambridge Univ. Press, Cambridge, 1994. 